PROCEEDINGS OF THE

AMERICAN MATHEMATICAL SOCIETY

Volume 139, Number 2, February 2011, Pages 511-520

S 0002-9939(2010)10497-5

Article electronically published on July 13, 2010

\title{
AN INDEX FORMULA FOR THE TWO VARIABLE JORDAN BLOCK
}

\author{
YUFENG LU, YIXIN YANG, AND RONGWEI YANG
}

(Communicated by Nigel J. Kalton)

\begin{abstract}
On Hardy space $H^{2}\left(\mathbb{D}^{2}\right)$ over the bidisk, let $\left(S_{z}, S_{w}\right)$ be the compression of the pair $\left(T_{z}, T_{w}\right)$ to the quotient module $H^{2}\left(\mathbb{D}^{2}\right) \ominus M$. In this paper, we obtain an index formula for $\left(S_{z}, S_{w}\right)$ when it is Fredholm. It is also shown that the evaluation operator $L(0)$ is compact on a Beurling type quotient module if and only if the corresponding inner function is a finite Blaschke product in $w$.
\end{abstract}

\section{INTRODUCTION}

In this paper, let $\mathbb{D}^{2}$ denote the open unit bidisk in $\mathbb{C}^{2}$ and let $\mathbb{T}^{2}$ denote the distinguished boundary of $\mathbb{D}^{2}$. The Hardy space $H^{2}\left(\mathbb{D}^{2}\right)$ is the closure of polynomials in $L^{2}\left(\mathbb{T}^{2}, \frac{1}{(2 \pi)^{2}} d \theta_{1} d \theta_{2}\right)$. On $H^{2}\left(\mathbb{D}^{2}\right)$, the Toeplitz operators $T_{z}$ and $T_{w}$ are unilateral shifts of infinity multiplicity. A closed subspace $M \subset H^{2}\left(\mathbb{D}^{2}\right)$ is called a submodule if it is invariant under the action of $T_{z}$ and $T_{w}$. The quotient space $H^{2}\left(\mathbb{D}^{2}\right) / M$, naturally identified with $H^{2}\left(\mathbb{D}^{2}\right) \ominus M$, is endowed with a $\mathbb{C}[z, w]$ - module structure by

$$
p \cdot f=p\left(S_{z}, S_{w}\right) f, \quad p \in \mathbb{C}[z, w], \quad f \in N,
$$

where $S_{z}=\left.P_{N} T_{z}\right|_{N}, S_{w}=\left.P_{N} T_{w}\right|_{N}, \mathbb{C}[z, w]$ is the polynomial ring in two variables, and $P_{N}$ stands for the orthogonal projection from $H^{2}\left(\mathbb{D}^{2}\right)$ onto $N$. Then $\left(S_{z}, S_{w}\right)$ is a pair of commuting contractions, which is called the two variable Jordan block. We also denote the compression of $T_{z}$ and $T_{w}$ on $M$ by $R_{z}$ and $R_{w}$, respectively, that is,

$$
R_{z}=\left.T_{z}\right|_{M}, \quad R_{w}=\left.T_{w}\right|_{M} .
$$

It is easy to see that $R=\left(R_{z}, R_{w}\right)$ is a pair of commuting isometries.

In the one variable Hardy space $H^{2}(\mathbb{D})$, the classical Beurling theorem implies that every submodule is of the form $M=\theta H^{2}(\mathbb{D})$ for some inner function $\theta$. In the one variable setting, the compression $S(\theta)=\left.P_{N} T_{z}\right|_{N}$ is called the Jordan block (cf. [Be $[\mathrm{SF}]$ ). Here $P_{N}$ is the orthogonal projection from $H^{2}(\mathbb{D})$ onto the associated quotient module $N$. It is well known that the index of $S(\theta)$ is always 0 . However, the structure of submodules of $H^{2}\left(\mathbb{D}^{2}\right)$ is far more complex. For instance, W. Rudin displayed two submodules in $[\mathrm{Ru}$, one which was of infinite rank, and the other

Received by the editors December 21, 2009 and, in revised form, March 5, 2010.

2010 Mathematics Subject Classification. Primary 47A13; Secondary 46E20.

Key words and phrases. Index formula, two variable Jordan block, evaluation operator, Hardy space over bidisk.

This research was supported by NSFC, grants no. 10671028 and 10971020 .

(C) 2010 American Mathematical Society 511

Reverts to public domain 28 years from publication 
which contained no nontrivial bounded functions. There has been a large amount of work on the operator theory and structure of Hardy submodules on the polydisk; see [CG], [CMY], DP, GM], Ya1-YYa and references therein.

If we denote $H^{2}(\mathbb{D}) \ominus z H^{2}(\mathbb{D})$ by $H^{2}(w)$ and $H^{2}(\mathbb{D}) \ominus w H^{2}(\mathbb{D})$ by $H^{2}(z)$, then

$$
H^{2}(w)=\operatorname{clos}\left\{\operatorname{span}\left\{w^{j}: j \geq 0\right\}\right\}, \quad H^{2}(z)=\operatorname{clos}\left\{\operatorname{span}\left\{z^{j}: j \geq 0\right\}\right\} .
$$

We define a left evaluation operator $L(0)$ from $H^{2}\left(\mathbb{D}^{2}\right)$ to $H^{2}(w)$ and a right evaluation operator $R(0)$ from $H^{2}\left(\mathbb{D}^{2}\right)$ to $H^{2}(z)$ by

$$
L(0) f(w)=f(0, w), \quad R(0)=f(z, 0), \quad f \in H^{2}\left(\mathbb{D}^{2}\right) .
$$

In Ya3, the third author showed that if $\phi$ is inner, then $L(0)$ is Hilbert-Schmidt on $H^{2}\left(\mathbb{D}^{2}\right) \ominus \phi H^{2}\left(\mathbb{D}^{2}\right)$ if and only if $\phi$ is a finite Blaschke product in $w$. In this paper, we will show that the condition is also a necessary and sufficient condition for the compactness of $L(0)$ on $H^{2}\left(\mathbb{D}^{2}\right) \ominus \phi H^{2}\left(\mathbb{D}^{2}\right)$; here we will take a different approach.

The core operator $C^{M}$ for a submodule $M \subset H^{2}\left(\mathbb{D}^{2}\right)$ is defined by

$$
C^{M}=I-R_{z} R_{z}^{*}-R_{w} R_{w}^{*}+R_{z} R_{w} R_{z}^{*} R_{w}^{*} .
$$

For convenience, we will suppress the " $M$ " in our writing. The core operator (also called the defect operator) has been been well studied on the bidisk or unit ball; see [Ar], GY], Guo and YYa]-Ya8. Let $E_{-1}(C)$ and $E_{1}(C)$ be the corresponding eigenspace for $C$. The index of $\left(S_{z}, S_{w}\right)$ can be determined with a mild condition. For example, it is indicated in GRS that if $M$ contains a bounded function that does not vanish at $(0,0)$, then $\operatorname{ind}\left(S_{z}, S_{w}\right)=0$. In Ya6 and Ya8, the author showed that if $C$ is a Hilbert-Schmidt operator, then $\left(R_{z}, R_{w}\right)$ and $\left(S_{z}, S_{w}\right)$ are both Fredholm, and $\operatorname{ind}\left(S_{z}, S_{w}\right)=-1-\operatorname{ind}\left(R_{z}, R_{w}\right)=\operatorname{dim} E_{1}(C)-E_{-1}(C)-1=0$. In this paper, we prove that if $\left(S_{z}, S_{w}\right)$ is Fredholm, then

$$
\operatorname{dim}((M \ominus z M) \cap(M \ominus w M))<\infty,
$$

and the index of $\left(S_{z}, S_{w}\right)$ also equals $\operatorname{dim} E_{1}(C)-E_{-1}(C)-1 .\left[R_{z}^{*}, R_{z}\right]\left[R_{w}^{*}, R_{w}\right]$ and $\left[R_{w}^{*}, R_{z}\right]$ are also important subjects in the study of Hardy submodule over the bidisk, and we will show that if $\left(S_{z}, S_{w}\right)$ is Fredholm, then $\left[R_{z}^{*}, R_{z}\right]\left[R_{w}^{*}, R_{w}\right]$ is compact if and only if $\left[R_{w}^{*}, R_{z}\right]$ is compact.

\section{Evaluation operators}

The evaluation operators, which turn functions of two variables into functions of one variable, serve as a bridge connecting the operator in $H^{2}\left(\mathbb{D}^{2}\right)$ to single operator theory. The evaluation operators also play an important role in the proof of Section 3.

We define a difference quotient operator $D_{z}$ from $H^{2}\left(\mathbb{D}^{2}\right)$ to itself by $D_{z} f(z, w)=$ $\frac{f(z, w)-L(0) f(w)}{z}, f \in H^{2}\left(\mathbb{D}^{2}\right)$. For any submodule $M, D_{z}$ maps $M \ominus z M$ into $N$ (cf. Ya3]). Without confusion, the restriction of $D_{z}$ on $M \ominus z M$ is also denoted by $D_{z}$. There are systemic studies on the evaluation operators and difference quotient operators; see [Ya3] and [Ya4].

In [Ya2, it is showed that $L(0)$ is always Hilbert-Schmidt on $M \ominus w M$; however, $L(0)$ may not be Hilbert-Schmidt on $M$. The following property gives a sufficient condition and may be of interest by itself.

Proposition 2.1. Let $M$ be a nonzero submodule in $H^{2}\left(\mathbb{D}^{2}\right)$. If $I-S_{z}^{*} S_{z}$ is in trace class, then the restriction of $L(0)$ on $M \ominus z M$ is not Hilbert-Schmidt. 
Proof. It is indicated in Ya3 that $I-S_{z}^{*} S_{z}=D_{z} D_{z}^{*}$. Hence, if $I-S_{z}^{*} S_{z}$ is in trace class, then $D_{z} D_{z}^{*}$ is in trace class.

Let $\left\{e_{i}: i=1,2, \cdots, l\right\}$ be the orthogonal basis for $N$, where $l=\operatorname{dim} N$ may be infinity, and let $\left\{f_{i}: i=1,2, \cdots, \infty\right\}$ be the orthogonal basis for $M \ominus z M$. Then

$$
D_{z}^{*} e_{i}=\sum_{j=1}^{\infty}\left\langle T_{z} e_{i}, f_{j}\right\rangle f_{j}
$$

Hence

$$
\begin{aligned}
\operatorname{Trace}\left(D_{z} D_{z}^{*}\right) & =\sum_{i=1}^{l}\left\langle D_{z} D_{z}^{*} e_{i}, e_{i}\right\rangle=\sum_{i=1}^{l}\left\|D_{z}^{*} e_{i}\right\|^{2} \\
& =\sum_{i=1}^{l} \sum_{j=1}^{\infty}\left|\left\langle T_{z} e_{i}, f_{j}\right\rangle\right|^{2} \\
& =\sum_{j=1}^{\infty}\left\|P_{N} T_{z}^{*} f_{j}\right\|^{2}=\sum_{j=1}^{\infty}\left\|\frac{f_{j}-L(0) f_{j}}{z}\right\|^{2} \\
& =\sum_{j=1}^{\infty}\left(\left\|f_{j}\right\|^{2}-\left\|L(0) f_{j}\right\|^{2}\right)<\infty .
\end{aligned}
$$

Since $\sum_{j=1}^{\infty}\left\|f_{j}\right\|^{2}=\operatorname{dim}(M \ominus z M)=\infty$ for any submodule $M$, it follows that $\left.L(0)\right|_{M \ominus z M}$ is not Hilbert-Schmidt. This completes the proof.

Example 1. If $\psi(w)$ is an analytic function with $\|\psi\|_{\infty}<1$ and $M=[z-\psi(w)]$, then on $H^{2}\left(\mathbb{D}^{2}\right) \ominus \psi H^{2}\left(\mathbb{D}^{2}\right), S_{z}=\psi\left(S_{w}\right)$, and therefore $\left\|S_{z}\right\| \leq\|\psi\|_{\infty}<1$. By Theorem 3.2 in Ya5], $I-S_{z}^{*} S_{z}$ is in trace class, and hence $L(0)$ is not HilbertSchmidt on $M$.

The compactness of $\left.L(0)\right|_{N}$ and $\left.R(0)\right|_{N}$ has a closed relationship with the essential normality of $N$; for example, see GW2. We will characterize the compactness of $L(0)$ on Beurling type quotient module completely. Let $M=\phi H^{2}\left(\mathbb{D}^{2}\right)$ be a Beurling type submodule, where $\phi$ is an inner function in $H^{2}\left(\mathbb{D}^{2}\right)$. Then $P_{M}=M_{\phi} M_{\phi}^{*}$ and $P_{N}=I-M_{\phi} M_{\phi}^{*}$ are the projections on $M$ and $N$, respectively. Let $P$ be the orthogonal projection from $L^{2}\left(\mathbb{T}^{2}\right)$ onto $H^{2}\left(\mathbb{D}^{2}\right)$. The Toeplitz operator with symbol $f \in L^{2}\left(\mathbb{T}^{2}\right)$ is defined by $T_{f}(h)=P(f h)$, and the Hankel operator $H_{f}$ is defined by $H_{f}(h)=(I-P)(f h)$, where $h \in H^{2}\left(\mathbb{D}^{2}\right)$. It is well known that

$$
T_{f g}-T_{f} T_{g}=H_{f}^{*} H_{g},
$$

for any $f, g \in L^{2}\left(\mathbb{T}^{2}\right)$.

Proposition 2.2. Let $M=\phi H^{2}\left(\mathbb{D}^{2}\right)$ be a Beurling type submodule and $N=$ $H^{2}\left(\mathbb{D}^{2}\right) \ominus \phi H^{2}\left(\mathbb{D}^{2}\right)$. Then $L(0)$ is compact on $N$ if and only if $\phi$ is a finite Blaschke product in $w$.

Proof. First we recall that for any $\lambda=\left(\lambda_{1}, \lambda_{2}\right) \in \mathbb{D}^{2}$, the normalized reproducing kernel of $H^{2}\left(\mathbb{D}^{2}\right)$ is

$$
k_{\lambda}(z, w)=\frac{K_{\lambda}(z, w)}{\left\|K_{\lambda}\right\|}=\frac{\sqrt{\left(1-\left|\lambda_{1}\right|^{2}\right)\left(1-\left|\lambda_{2}\right|^{2}\right)}}{\left(1-\overline{\lambda_{1}} z\right)\left(1-\overline{\lambda_{2}} w\right)},
$$


and $k_{\lambda_{2}}(w)=\frac{K_{\lambda_{2}}(w)}{\left\|K_{\lambda_{2}}\right\|}=\frac{\sqrt{\left(1-\left|\lambda_{2}\right|^{2}\right)}}{1-\overline{\lambda_{2}} w}$ is the normalized reproducing kernel of $H^{2}(w)$.

Now assume that $L(0) P_{N}$ is compact. By Lemma 1.2.1 in [Ya3], $L\left(\lambda_{1}\right) P_{N}$ is compact for any $\lambda_{1} \in \mathbb{D}$; hence

$$
\lim _{\lambda_{2} \rightarrow \mathbb{T}}\left\|L\left(\lambda_{1}\right) P_{N} k_{\lambda}\right\|=0
$$

Since $P_{N} k_{\lambda}=k_{\lambda}-M_{\phi} M_{\phi}^{*} k_{\lambda}=(1-\overline{\phi(\lambda)} \phi) k_{\lambda}$, then $L\left(\lambda_{1}\right) P_{N} k_{\lambda}=\frac{1}{\sqrt{1-\left|\lambda_{1}\right|^{2}}}(1-$ $\left.\overline{\phi(\lambda)} \phi\left(\lambda_{1}, w\right)\right) k_{\lambda_{2}}$. Therefore,

$$
\begin{aligned}
\left\|L\left(\lambda_{1}\right) P_{N} k_{\lambda}\right\|^{2} & =\frac{1}{1-\left|\lambda_{1}\right|^{2}}\left\langle\left(1-\overline{\phi(\lambda)} \phi\left(\lambda_{1}, w\right)\right) k_{\lambda_{2}},\left(1-\overline{\phi(\lambda)} \phi\left(\lambda_{1}, w\right)\right) k_{\lambda_{2}}\right\rangle \\
& =\frac{1}{1-\left|\lambda_{1}\right|^{2}}\left(1-2|\phi(\lambda)|^{2}+|\phi(\lambda)|^{2}\left\langle M_{\phi\left(\lambda_{1}, w\right)}^{*} M_{\phi\left(\lambda_{1}, w\right)} k_{\lambda_{2}}, k_{\lambda_{2}}\right\rangle\right) .
\end{aligned}
$$

From formula (2.1), we have

$$
M_{\phi\left(\lambda_{1}, w\right)}^{*} M_{\phi\left(\lambda_{1}, w\right)}=M_{\phi\left(\lambda_{1}, w\right)} M_{\phi\left(\lambda_{1}, w\right)}^{*}+H_{\overline{\phi\left(\lambda_{1}, w\right)}}^{*} H_{\overline{\phi\left(\lambda_{1}, w\right)}} .
$$

Then it follows that

$$
\left\langle M_{\phi\left(\lambda_{1}, w\right)}^{*} M_{\phi\left(\lambda_{1}, w\right)} k_{\lambda_{2}}, k_{\lambda_{2}}\right\rangle=|\phi(\lambda)|^{2}+\left\|H_{\overline{\phi\left(\lambda_{1}, w\right)}} k_{\lambda_{2}}\right\|^{2} .
$$

Hence

$$
\left\|L\left(\lambda_{1}\right) P_{N} k_{\lambda}\right\|^{2}=\frac{1}{1-\left|\lambda_{1}\right|^{2}}\left(\left(1-|\phi(\lambda)|^{2}\right)^{2}+\left\|H_{\overline{\phi\left(\lambda_{1}, w\right)}} k_{\lambda_{2}}\right\|^{2}\right),
$$

which together with (2.2) gives us that

$$
\lim _{\lambda_{2} \rightarrow \mathbb{T}}|\phi(\lambda)|=1, \quad \lambda_{1} \in \mathbb{D} .
$$

It follows that there is a subset $E$ of $\mathbb{T}$ with measure 1 such that for any $\lambda_{1} \in \mathbb{D}$,

$$
\left|\phi\left(\lambda_{1}, w\right)\right|=1, \quad w \in E
$$

and hence $\phi(\cdot, w)$ is a constant function with module 1 for every $w \in E$.

Let

$$
\phi(z, w)=\sum_{i=0}^{\infty} \phi_{i}(w) z^{i}, \quad \phi_{i} \in H^{2}(w) .
$$

Then for every $w \in E$ and $k=1,2, \ldots$, we have

$$
\left.\frac{\partial^{k} \phi(z, w)}{\partial z^{k}}\right|_{z=0}=\phi_{k}(w)=0,
$$

and hence $\phi_{k}=0$, i.e., $\phi=\phi(w)$. It is easy to check that $H^{2}(w) \ominus \phi H^{2}(w) \subset$ $H^{2}\left(\mathbb{D}^{2}\right) \ominus \phi H^{2}\left(\mathbb{D}^{2}\right)$ and $L(0)$ is an isometry acting on it. The compactness of $L(0)$ implies that $H^{2}(w) \ominus \phi H^{2}(w)$ is finite dimensional, which is possible only if $\phi$ is a finite Blaschke product in $w$.

On the other hand, if $\phi$ is a finite Blaschke product in $w$, by Corollary 4.2 .2 in Ya3 $L(0)$ is Hilbert-Schmidt on $N$. This completes the proof. 


\section{AN INDEX FORMULA}

In this section, we will prove that if $\left(S_{z}, S_{w}\right)$ is Fredholm, then

$$
\operatorname{dim}((M \ominus z M) \cap(M \ominus w M))<\infty,
$$

and we will give an index formula for $\left(S_{z}, S_{w}\right)$, i.e., $\operatorname{ind}\left(S_{z}, S_{w}\right)=\operatorname{dim} E_{1}(C)-$ $E_{-1}(C)-1$. First, let us recall some notation and useful tools.

Koszul complex. For every pair of commutating operators $A=\left(A_{1}, A_{2}\right)$ on a Hilbert space $H$, there is a short sequence

$$
0 \rightarrow H \stackrel{d_{1}}{\rightarrow} H \oplus H \stackrel{d_{2}}{\longrightarrow} H \rightarrow 0,
$$

where

$$
d_{1} x=\left(-A_{2} x, A_{1} x\right), \quad d_{2}(x, y)=A_{1} x+A_{2} y, \quad x, y \in H .
$$

It is easy to check that

$$
d_{2} d_{1}=0 .
$$

The tuple $A=\left(A_{1}, A_{2}\right)$ is said to be Fredholm if $d_{1}$ and $d_{2}$ both have closed range and

$$
\operatorname{dim}\left(\operatorname{Ker}\left(d_{1}\right)\right)+\operatorname{dim}\left(\operatorname{Ker}\left(d_{2}\right) \ominus d_{1}(H)\right)+\operatorname{dim}\left(H \ominus d_{2}(H \oplus H)\right)<\infty .
$$

It is well known (cf. [Cu] ) that the tuple $A=\left(A_{1}, A_{2}\right)$ is Fredholm if and only if

$$
\widehat{A}=\left(\begin{array}{cc}
A_{1} & A_{2} \\
-A_{2}^{*} & A_{1}^{*}
\end{array}\right)
$$

is Fredholm on $H \oplus H$, and in this case $\operatorname{ind}\left(A_{1}, A_{2}\right)=i n d \widehat{A}$.

We refer the reader to $\mathrm{Cu}$ for more detailed discussions of Fredholm complexes for $n$-tuples.

Two defect operators for $S=\left(S_{z}, S_{w}\right)$ are defined by

$$
\Delta_{S}=I-S_{z}^{*} S_{z}-S_{w}^{*} S_{w}+S_{z}^{*} S_{w}^{*} S_{z} S_{w}
$$

and

$$
\Delta_{S^{*}}=I-S_{z} S_{z}^{*}-S_{w} S_{w}^{*}+S_{z} S_{w} S_{z}^{*} S_{w}^{*}
$$

$C$ and $\Delta_{S}$ are useful tools in the study of Hardy submodules, and we refer the reader to $\mathrm{GY}$, Ya7] and Ya8, for more details.

Let $\eta$ denote the function $P_{N} 1$. If $M$ is nontrivial, then $1 \notin M$, i.e., $\eta \neq 0$. It is indicated in [Ya7] that $\Delta_{S^{*}}$ is a rank one operator, and in fact $\Delta_{S^{*}}=\eta \otimes \eta$. We write $Z(M)=\left\{(\lambda, \mu) \in \mathbb{D}^{2} \mid f(\lambda, \mu)=0\right.$, for all $\left.f \in M\right\}$.

Lemma 3.1. Let $M$ be a nontrivial submodule in $H^{2}\left(\mathbb{D}^{2}\right)$. If $S=\left(S_{z}, S_{w}\right)$ is Fredholm, then $\operatorname{dim}((M \ominus z M) \cap(M \ominus w M)) \leq \operatorname{dimker} \widehat{S}+1<\infty$. More precisely, if $(0,0) \in Z(M)$, then $\operatorname{dim}((M \ominus z M) \cap(M \ominus w M))=\operatorname{dimker} \widehat{S}$, and if $(0,0) \notin Z(M)$, then $\operatorname{dim}((M \ominus z M) \cap(M \ominus w M))=\operatorname{dimker} \widehat{S}+1$.

Proof. If $S=\left(S_{z}, S_{w}\right)$ is Fredholm, then

$$
\widehat{S}=\left(\begin{array}{cc}
S_{z} & S_{w} \\
-S_{w}^{*} & S_{z}^{*}
\end{array}\right)
$$

is Fredholm on $N \oplus N$. It is easy to check that

$$
k e r \widehat{S}=\left\{(f, g) \in N \oplus N \mid z f+w g \in M,-\bar{w} f+\bar{z} g \perp H^{2}\left(\mathbb{D}^{2}\right)\right\} .
$$


It follows from Ya8, that there is a bounded below map $A$ from $k e r \widehat{S}$ into $(M \ominus$ $z M) \cap(M \ominus w M)$ defined by

$$
A(f, g)=P_{M}(I+L(0)+R(0))(z f+w g),
$$

where $(f, g) \in k e r \widehat{S}$. Therefore, for any $h \in((M \ominus z M) \cap(M \ominus w M)) \ominus \operatorname{Ran} A$, $(f, g) \in k e r \widehat{S}$, we have

$$
\begin{aligned}
\langle h, A(f, g)\rangle & =\langle h,(I+L(0)+R(0))(z f+w g)\rangle \\
& =\langle h, z f+z R(0) f\rangle+\langle h, w g+w L(0) g\rangle \\
& =\left\langle T_{z}^{*} h+R(0) T_{z}^{*} h, f\right\rangle+\left\langle T_{w}^{*} h+L(0) T_{w}^{*} h, g\right\rangle \\
& =0 .
\end{aligned}
$$

Note that $T_{z}^{*} h \in N$ and $T_{w}^{*} h \in N$. It follows that

$$
\left(T_{z}^{*} h+P_{N} R(0) T_{z}^{*} h, T_{w}^{*} h+P_{N} L(0) T_{w}^{*} h\right) \in(k e r \widehat{S})^{\perp} .
$$

In view of the Fredholmness of $\widehat{S}$ on $N \oplus N$, we conclude that $\operatorname{Ran} \widehat{S}$ is closed and so is $\operatorname{Ran} \widehat{S}^{*}$. Hence there exists $(f, g) \in N \oplus N$ such that

$$
\widehat{S}^{*}(f, g)=\left(T_{z}^{*} h+P_{N} R(0) T_{z}^{*} h, T_{w}^{*} h+P_{N} L(0) T_{w}^{*} h\right),
$$

i.e.,

$$
\begin{aligned}
& S_{z}^{*} f-S_{w} g=T_{z}^{*} h+P_{N} R(0) T_{z}^{*} h, \\
& S_{w}^{*} f+S_{z} g=T_{w}^{*} h+P_{N} L(0) T_{w}^{*} h .
\end{aligned}
$$

If $h=0$, then $S_{z}^{*} f-S_{w} g=0$ and $S_{w}^{*} f+S_{z} g=0$. Since $S_{z}$ commutes with $S_{w}$, we have $S_{z} S_{z}^{*} f+S_{w} S_{w}^{*} f=0$; that is, $f \in \operatorname{ker} S_{z}^{*} \cap \operatorname{ker} S_{w}^{*}$. Indeed, we have a linear map $T$ from $((M \ominus z M) \cap(M \ominus w M)) \ominus \operatorname{Ran} A$ to the quotient space $N /\left(\operatorname{ker} S_{z}^{*} \cap k e r S_{w}^{*}\right)$ defined by

$$
T h=f+\operatorname{ker} S_{z}^{*} \cap \operatorname{ker} S_{w}^{*} .
$$

We now check that $T$ is injective. If $f+\operatorname{ker} S_{z}^{*} \cap \operatorname{ker} S_{w}^{*}=0$, i.e., $f \in \operatorname{ker} S_{z}^{*} \cap$ $k e r S_{w}^{*}$, we have

$$
\begin{aligned}
-S_{w} g & =T_{z}^{*} h+P_{N} R(0) T_{z}^{*} h, \\
S_{z} g & =T_{w}^{*} h+P_{N} L(0) T_{w}^{*} h .
\end{aligned}
$$

It yields that

$$
\begin{aligned}
& \frac{h-h(0, w)}{z}+\frac{h(z, 0)-h(0,0)}{z}+w g \in M, \\
& \frac{h-h(z, 0)}{w}+\frac{h(0, w)-h(0,0)}{w}-z g \in M,
\end{aligned}
$$

i.e.,

$$
\begin{aligned}
& h-h(0, w)+h(z, 0)-h(0,0)+z w g \in z M, \\
& h-h(z, 0)+h(0, w)-h(0,0)-z w g \in w M .
\end{aligned}
$$

Adding the left parts of (3.3) and (3.4), we obtain

$$
2 h-2 h(0,0) \in M \text {. }
$$

Then $h(0,0) \in M$, which in turn implies that $h(0,0)=0$, since $M$ is a nontrivial submodule. Note that $h \in(M \ominus z M) \cap(M \ominus w M)$, and it follows from (3.3) and (3.4) that

$$
\langle h-h(0, w)+h(z, 0)+z w g, h\rangle=0
$$


and

$$
\langle h-h(z, 0)+h(0, w)-z w g, h\rangle=0,
$$

i.e.,

$$
\|h\|^{2}-\|L(0) h\|^{2}+\|R(0) h\|^{2}+\langle z w g, h\rangle=0
$$

and

$$
\|h\|^{2}-\|R(0) h\|^{2}+\|L(0) h\|^{2}-\langle z w g, h\rangle=0 .
$$

Adding (3.5) and (3.6), we obtain $2\|h\|^{2}=0$. Then $h=0$ and $T$ is injective.

Now we prove that the dimension of RanT is less than 1. Adding (3.1) multiplied by $S_{z}$ with (3.2) multiplied by $S_{w}$, we have

$$
\left(S_{z} S_{z}^{*}+S_{w} S_{w}^{*}\right) f=S_{z} T_{z}^{*} h+S_{z} P_{N} R(0) T_{z}^{*} h+S_{w} T_{w}^{*} h+S_{w} P_{N} L(0) T_{w}^{*} h .
$$

Calculating the four items on the right-hand side of (3.7) one by one, we have

$$
\begin{aligned}
& S_{z} T_{z}^{*} h=S_{z} \frac{h-h(0, w)}{z}=-P_{N} h(0, w), \\
& S_{z} P_{N} R(0) T_{z}^{*} h=S_{z} R(0) T_{z}^{*} h \\
& =S_{z} \frac{h(z, 0)-h(0,0)}{z} \\
& =P_{N} h(z, 0)-h(0,0) \eta .
\end{aligned}
$$

Similarly, we have $S_{w} T_{w}^{*} h=S_{w} \frac{h-h(z, 0)}{w}=-P_{N} h(z, 0)$ and $S_{w} P_{N} L(0) T_{w}^{*} h=$ $P_{N} h(0, w)-h(0,0) \eta$. Hence

$$
\left(S_{z} S_{z}^{*}+S_{w} S_{w}^{*}\right) f=-2 h(0,0) \eta .
$$

Note that $\Delta_{S^{*}}=I-S_{z} S_{z}^{*}-S_{w} S_{w}^{*}+S_{z} S_{w} S_{z}^{*} S_{w}^{*}=\eta \otimes \eta$, and it follows that $\left(I+S_{z} S_{w} S_{z}^{*} S_{w}^{*}\right) f=(\eta \otimes \eta) f-2 h(0,0) \eta$. Since $I+S_{z} S_{w} S_{z}^{*} S_{w}^{*}$ is an invertible operator, we obtain that $\operatorname{dimRanT} \leq 1$, and thus $\operatorname{dim}(((M \ominus z M) \cap(M \ominus w M)) \ominus$ $\operatorname{Ran} A) \leq 1$. That is,

$$
\operatorname{dim}((M \ominus z M) \cap(M \ominus w M)) \leq \operatorname{dimker} \widehat{S}+1<\infty .
$$

If $(0,0) \in Z(M)$, then for every $h \in M, h(0,0)=0$. By the previous arguments, for any $h \in((M \ominus z M) \cap(M \ominus w M)) \ominus \operatorname{Ran} A, h=0$, and hence $\operatorname{dim}((M \ominus z M) \cap$ $(M \ominus w M))=\operatorname{dimRan} A=\operatorname{dimker} \widehat{S}$.

Note that $(0,0) \notin Z(M)$ if and only if $1 \notin N$. We claim that $P_{M} 1 \notin \operatorname{Ran} A$; otherwise, there is $h=z f+w g \in M$ such that $P_{M} 1=P_{M}(I+L(0)+R(0)) h$, where $(f, g) \in k e r \widehat{S}$. Then $\left\langle h, P_{M} 1\right\rangle=\left\langle h, P_{M}(I+L(0)+R(0)) h\right\rangle$, i.e., $\|h\|^{2}+$ $\|L(0) h\|^{2}+\|R(0) h\|^{2}=0$, which implies that $h=0$ and hence $P_{M} 1=0$, which contradicts $(0,0) \notin Z(M)$. It is obvious that $P_{M} 1 \in(M \ominus z M) \cap(M \ominus w M)$. Then the orthogonal projection of $P_{M} 1$ from $(M \ominus z M) \cap(M \ominus w M)$ into $((M \ominus z M) \cap$ $(M \ominus w M)) \ominus \operatorname{Ran} A$ is not zero, that is, $\operatorname{dim}(((M \ominus z M) \cap(M \ominus w M)) \ominus \operatorname{Ran} A) \geq 1$. Combining the previous results, we have $\operatorname{dim}((M \ominus z M) \cap(M \ominus w M))=\operatorname{dimRan} A+$ $1=\operatorname{dimker} \widehat{S}+1$.

This completes the proof of Lemma 3.1. 
Theorem 3.2. Let $M$ be a submodule in $H^{2}\left(\mathbb{D}^{2}\right)$. If $S=\left(S_{z}, S_{w}\right)$ is Fredholm, then $\operatorname{ind}\left(S_{z}, S_{w}\right)=\operatorname{dim} E_{1}(C)-\operatorname{dim} E_{-1}(C)-1$.

Proof. Curto's lemma implies that $\operatorname{ind}\left(S_{z}, S_{w}\right)=$ dimker $\widehat{S}-$ dimker $\widehat{S}^{*}$. If $(0,0) \in$ $Z(M)$, then $1 \in N$, which implies that $1 \in \operatorname{ker} S_{z}^{*} \cap \operatorname{ker} S_{w}^{*}$. Since $\operatorname{ker} S_{z}^{*} \cap \operatorname{ker} S_{w}^{*} \subset \mathbb{C}$, we have $\operatorname{dim}\left(\operatorname{ker} S_{z}^{*} \cap \operatorname{ker} S_{w}^{*}\right)=1$ and $\operatorname{dimker} \widehat{S}^{*}=1+\operatorname{dim}\left(\operatorname{ker} S_{z} \cap \operatorname{ker} S_{w}\right)$. If $(0,0) \notin Z(M)$, then $\operatorname{ker} S_{z}^{*} \cap \operatorname{ker} S_{w}^{*}=\{0\}$ and hence $\operatorname{dimker} \widehat{S}^{*}=\operatorname{dim}\left(\operatorname{ker} S_{z} \cap\right.$ $\left.k e r S_{w}\right)$. By Lemma 3.1 we have, in any case, that

$$
\operatorname{ind}\left(S_{z}, S_{w}\right)=\operatorname{dim}((M \ominus z M) \cap(M \ominus w M))-\operatorname{dim}\left(\operatorname{ker} S_{z} \cap \operatorname{ker} S_{w}\right)-1 .
$$

By Corollary 3.4 in [GY], $E_{1}(C)=(M \ominus z M) \cap(M \ominus w M)$ and $E_{-1}(C)=(z M \cap$ $w M) \ominus z w M$. It is indicated in GW1 and Ya1 that $\operatorname{dim}\left(\operatorname{ker} S_{z} \cap \operatorname{ker} S_{w}\right)=$ $\operatorname{dim}((z M \cap w M) \ominus z w M)$, and this completes the proof of Theorem 3.2.

For a submodule $M$, we define two operators $F_{z}$ on $M \ominus z M$ and $F_{w}$ on $M \ominus w M$ by

$$
F_{z} f=\left[R_{z}^{*}, R_{z}\right] w f, \quad F_{w} g=\left[R_{w}^{*}, R_{w}\right] z f,
$$

where $f \in M \ominus z M, g \in M \ominus w M$, respectively.

Since $F_{z}$ and $F_{w}$ display parallel properties, we just list some properties of $F_{z}$; more details can be found in Ya4.

Lemma 3.3 ([पa4]). Let $M$ be a submodule. Then the following hold:

(1) $\operatorname{RanF}_{z}=(z M+w M) \ominus z M, \operatorname{kerF}_{z}=z\left(\operatorname{ker} S_{z} \cap \operatorname{ker} S_{w}\right)$.

(2) If $f \in M \ominus z M$, then $f-F_{z}^{*} F_{z} f=\left[R_{w}^{*}, R_{z}\right]\left[R_{z}^{*}, R_{w}\right] f, f-F_{z} F_{z}^{*} f=$ $\left[R_{z}^{*}, R_{z}\right]\left[R_{w}^{*}, R_{w}\right] f$.

(3) For every $f \in M \ominus z M,\left[R_{w}^{*}, R_{w}\right]\left[R_{z}^{*}, R_{z}\right] F_{z} f=-F_{w}\left[R_{z}^{*}, R_{w}\right] f$, and for every $f \in M \ominus w M,\left[R_{z}^{*}, R_{z}\right]\left[R_{w}^{*}, R_{w}\right] F_{w} f=-F_{z}\left[R_{w}^{*}, R_{z}\right] f$.

Corollary 3.4. Let $M$ be a submodule in $H^{2}\left(\mathbb{D}^{2}\right), N=H^{2}\left(\mathbb{D}^{2}\right) \ominus M$. If $\left(S_{z}, S_{w}\right)$ is Fredholm, then $\left[R_{z}^{*}, R_{z}\right]\left[R_{w}^{*}, R_{w}\right]$ is compact if and only if $\left[R_{w}^{*}, R_{z}\right]$ is compact.

Proof. If $\left(S_{z}, S_{w}\right)$ is Fredholm, combining Lemma 3.1 and Lemma 3.3 (1), we have $\operatorname{dim}\left((M \ominus z M) \ominus \overline{\operatorname{RanF}_{z}}\right)=\operatorname{dim}((M \ominus z M) \cap(M \ominus w M))<\infty$, and $\operatorname{dimker} F_{z}=$ $\operatorname{dim}\left(\operatorname{ker}\left(S_{z}\right) \cap \operatorname{ker} S_{w}\right)<\infty$.

If $\left[R_{w}^{*}, R_{z}\right]$ is compact, then by Lemma $3.3(2), R a n F_{z}$ is closed and then $F_{z}$ is Fredholm. Lemma $3.3(3)$ implies that $\left[R_{z}^{*}, R_{z}\right]\left[R_{w}^{*}, R_{w}\right]$ is compact.

If $\left[R_{z}^{*}, R_{z}\right]\left[R_{w}^{*}, R_{w}\right]$ is compact, the parallel properties of $F_{w}$ and the above arguments imply that $\left[R_{w}^{*}, R_{z}\right]$ is compact.

This completes the proof of Corollary 3.4.

The closedness of $z M+w M$ is worth studying for many reasons. We have been unable to prove whether the Fredholmness of $\left(S_{z}, S_{w}\right)$ implies the closedness of $z M+w M$. If so, then the Fredholmness of $\left(S_{z}, S_{w}\right)$ implies the Fredholmness of $\left(R_{z}, R_{w}\right)$ and the Fredholmness of $F_{z} ;$ moreover, $\operatorname{ind}\left(S_{z}, S_{w}\right)=-1-\operatorname{ind}\left(R_{z}, R_{w}\right)=$ $-1-i n d F_{z}$. 


\section{ACKNOWLEDGEMENT}

The authors would like to express their sincere thanks to the referee, whose comments considerably improved the original version of the paper.

\section{REFERENCES}

[Ar] W. Arveson, The curvature invariant of a Hilbert module over $\mathbb{C}\left[z_{1}, z_{2}, \cdots, z_{n}\right]$, J. Reine Angew Math. 522(2000), 173-236. MR.1758582(2003a:47013)

[Be] K. Bercovici, Operator theory and arithmetic in $H^{\infty}$, Mathematical Surveys and Monographs, No. 26, Amer. Math. Soc., 1988, Providence, RI. MR 954383 (90e:47001)

[CG] X. Chen and K. Guo, Analytic Hilbert modules, Chapman Hall/CRC, Boca Raton, FL, 2003. MR 1988884 (2004d:47024)

[CMY] R. Curto, P. Muhly and K. Yan, The $C^{*}$-algebra of a homogeneous ideal in two variables is type I, Current topics in operator algebras (Nara, 1990), 130-136, World Sci. Publishing, River Edge, NJ, 1991. MR:1193935 (93h:47055)

$[\mathrm{Cu}] \quad$ R. Curto, Fredholm and invertible $n$-tuples of operators. The deformation problem. Trans. Amer. Math. Soc. 266(1981), no. 1, 129-159. MR613789 (82g:47010)

[DP] R. G. Douglas and V. I. Paulsen, Hilbert modules over function algebras, Longman Research Notes in Mathematics, Vol. 217, Longman, Harlow, NY, 1989. MR:1028546 (91g:46084)

[GM] P. Ghatage and V. Mandrekar, On Beurling type invariant subspaces of $L^{2}\left(T^{2}\right)$ and their equivalence, J. Oper. Theory 20(1988), no. 1, 83-89. MR972182 (90i:47005)

[GRS] D. Greene, S. Richter and C. Sundberg, On the index of invariant subspaces in spaces of analytic functions of several complex variables, J. Reine. Angew. Math. 587(2005), 49-76. MR2186975 (2006i:47013)

[Guo] K. Guo, Defect operators, defect functions and defect indices for analytic submodules, J. Funct. Anal. 213(2004), 380-411. MR2078631 (2005e:47016)

[GW1] K. Guo and P. Wang, Defect operators and Fredholmness for Toeplitz pairs with inner symbols, J. Oper. Theory 58:2(2007), 251-268. MR.2358530(2008i:47057)

[GW2] K. Guo and P. Wang, Essentially normal Hilbert modules and $K$-homology. III. Homogenous quotient modules of Hardy modules on the bidisk, Sci. China Ser. A 50(2007), 387411. MR 2334557(2008j:47006)

[GY] K. Guo and R. Yang, The core function of Hardy submodules over the bidisk, Indiana Univ. Math. J. 53(2004), 205-222. MR2048190 (2005m:46048)

$[\mathrm{Ru}]$ W. Rudin, Function theory in polydiscs, Benjamin, New York, 1969. MR0255841(41:501)

[SF] B. Sz.-Nagy and C. Foiaş, Harmonic analysis of operators on Hilbert space, North-Holland, Amsterdam; American Elsevier, New York; Akad. Kiadó, Budapest, 1970. MR0275190 $(43: 947)$

[Ya1] R. Yang, BCL index and Fredholm tuples, Proc. Amer. Math. Soc. 127(1999), no. 8, 2385-2393. MR 1605949 (99j:47013)

[Ya2] R. Yang, The Berger-Shaw theorem in the Hardy module over the bidisk, J. Oper. Theory 42(1999), no. 2, 379-404. MR1717024 (2000h:47040)

[Ya3] R. Yang, Operator theory in the Hardy space over the bidisk. II, Int. Equ. Oper. Theory 42(2002), 99-124. MR.1866878 (2002m:47007)

[Ya4] R. Yang, Operator theory in the Hardy space over the bidisk. III, J. Funct. Anal. 186(2001), 521-545. MR 1864831 (2002m:47008)

[Ya5] R. Yang, On two-variable Jordan blocks, Acta Sci. Math. (Szeged) 69(2003), 739-754. MR 2034205 (2004j:47011)

[Ya6] R. Yang, Beurling's phenomenon in two variables, Integr. Equ. Oper. Theory 48(2004), no. 3, 411-423. MR2038510 (2004j:46038)

[Ya7] R. Yang, The core operator and congruent submodules, J. Funct. Anal. 228(2005), 469486. MR2175415 (2006e:47015)

[Ya8] R. Yang, On two variable Jordan block. II, Int. Equ. Oper. Theory 56(2006), 431-449. MR2270846 (2007i:47006) 
School of Mathematical Sciences, Dalian University of Technology, Dalian 116024, People's Republic of China

E-mail address: lyfdlut1@yahoo.com.cn

School of Mathematical Sciences, Dalian University of Technology, Dalian 116024, People's Republic of China

E-mail address: yangyx314272@yahoo.com.cn

Department of Mathematical and Statistics, State University of New York at Albany, Albany, New York 12222

E-mail address: ryang@math.albany.edu 